Ferrata Storti Foundation

\title{
Glucocorticoids and selumetinib are highly synergistic in RAS pathway-mutated childhood acute lymphoblastic leukemia through upregulation of BIM
} Jackson, ${ }^{1}$ Dino Masic, ${ }^{1}$ Gareth Veal, ${ }^{1}$ Chris Halsey, ${ }^{2}$ David R. Newell, ${ }^{1}$ Josef Vormoor $^{1,3}$ and Julie A.E. Irving ${ }^{1}$

Haematologica 2019

Volume 104(9):1804-1811

${ }^{1}$ Newcastle Cancer Centre at the Northern Institute for Cancer Research, Newcastle University, Newcastle upon Tyne; ${ }^{2}$ Wolfson Wohl Cancer Research Centre, Institute of Cancer Sciences, College of Medical, Veterinary and Life Sciences, University of Glasgow, Glasgow and ${ }^{3}$ Great North Children's Hospital, Newcastle upon Tyne Hospitals NHS Foundation Trust, Newcastle upon Tyne, UK

\section{ABSTRACT}

$\mathrm{N}$ ew drugs are needed for the treatment of relapsed acute lymphoblastic leukemia and preclinical evaluation of the MEK inhibitor, selumetinib, has shown that this drug has excellent activity in those leukemias with RAS pathway mutations. The proapoptotic protein, BIM is pivotal in the induction of cell death by both selumetinib and glucocorticoids, suggesting the potential for synergy. Thus, combination indices for dexamethasone and selumetinib were determined in RAS pathway-mutated acute lymphoblastic leukemia primagraft cells in vitro and were indicative of strong synergism (combination index $<0.2 ; \mathrm{n}=5$ ). Associated pharmacodynamic assays were consistent with the hypothesis that the drug combination enhanced BIM upregulation over that achieved by a single drug alone. Dosing of dexamethasone and selumetinib singly and in combination in mice engrafted with primary-derived RAS pathway-mutated leukemia cells resulted in a marked reduction in spleen size which was significantly greater with the drug combination. Assessment of the central nervous system leukemia burden showed a significant reduction in the drug-treated mice, with no detectable leukemia in those treated with the drug combination. These data suggest that a selumetinib-dexamethasone combination may be highly effective in RAS pathway-mutated acute lymphoblastic leukemia. An international phase I/II clinical trial of dexamethasone and selumetinib (Seludex trial) is underway in children with multiply relapsed/refractory disease.

doi:10.3324/haematol.2017.185975

Check the online version for the most updated information on this article, online supplements, and information on authorship \& disclosures: www.haematologica.org/content/104/9/1804

(C)2019 Ferrata Storti Foundation

Material published in Haematologica is covered by copyright. All rights are reserved to the Ferrata Storti Foundation. Use of published material is allowed under the following terms and conditions:

https://creativecommons.org/licenses/by-nc/4.0/legalcode. Copies of published material are allowed for personal or internal use. Sharing published material for non-commercial purposes is subject to the following conditions: https://creativecommons.org//icenses/by-nc/4.0/legalcode, sect. 3. Reproducing and sharing published material for commercial purposes is not allowed without permission in writing from the publisher.

\section{Introduction}

Progress in the treatment of childhood acute lymphoblastic leukemia has been exceptional and, using contemporary regimens, sustained remission is achievable in almost $90 \%$ of children. ${ }^{1,2}$ However, the outcome of children who relapse is much poorer and remains a frequent cause of death in children with cancer. ${ }^{3.5}$ Since further intensification with traditional agents is often associated with significant toxicity and limited success, new therapies are clearly needed. One promising avenue that may deliver novel drugs comes from our previous work showing that mutation in genes which activate the Ras/Raf/Mek/Erk pathway, such as NRAS, KRAS, FLT3, and PTPN11, are highly prevalent in relapsed ALL and, importantly, mutated ALL cells are differentially sensitive to the MEK inhibitor, selumetinib (AZD6244, ARRY-142886) ${ }^{6.8}$ In contrast, RAS pathway wildtype ALL cells were insensitive to MEK inhibition, both in vitro and in vivo. ${ }^{6}$ In the IBFMREZ2002 clinical trial for relapsed ALL, RAS pathway mutations were associated with high-risk features such as early relapse, central nervous system (CNS) disease and chemo-resistance and a poorer overall survival was seen in patients with KRAS mutations. ${ }^{6}$ In 
the UKALLR3 trial, a poorer survival was seen in children with NRAS mutations.' Thus, this genetic subtype of relapsed ALL clearly warrants exploratory therapies.

The Ras/Raf/Mek/Erk cascade regulates diverse cellular functions, including cell proliferation, survival, differentiation, angiogenesis and migration, and is deregulated in numerous cancers, including ALL. ${ }^{9-13}$ Classic activation is initiated by ligand binding to receptor tyrosine kinases at the cell surface and via Ras, then Raf activates MEK1/2 which has restricted substrate specify for extracellular signal-regulated kinase 1 and 2 (Erk). ERK is a potent kinase with over 200 nuclear and cytoplasmic substrates including transcription factors such as the ETS family and proteins involved in the apoptotic machinery, such as the proapoptotic BIM. Phosphorylation of the predominant form of BIM (BIM EL $_{\text {L }}$ by ERK1/2, targets it for ubiquitination and proteasomal degradation and may also directly hinder its interactions with $\mathrm{Bax}^{14,15}$ and selumetinib-induced apoptosis is associated with BIM induction. ${ }^{16}$

Relapsed ALL is generally more drug resistant than newly diagnosed disease and despite the use of more intensive chemotherapeutic regimens at ALL relapse, there are lower rates of complete remission and end-ofinduction negativity for minimal residual disease., ${ }^{2,3}$ Assessment of in vitro drug sensitivity of primary ALL samples has shown that blasts at relapse are significantly more resistant to many of the drugs used in upfront treatment protocols, with the highest level of drug resistance seen to glucocorticoids. ${ }^{17,18}$ Glucocorticoids, such as dexamethasone, are pivotal agents in the treatment of all lymphoid malignancies because of their ability to specifically induce apoptosis in developing lymphocytes and induction of pro-apoptotic BIM is key to this effect. ${ }^{19}$ Thus, BIM is a common effector in both selumetinib- and dexamethasone-induced apoptosis, suggesting the potential for synergy. In addition, glucocorticoid resistance in ALL has been associated with enhanced activation of the pathway and its inhibition has led to glucocorticoid re-sensitization. ${ }^{20-22}$ These effects may be more pronounced in the context of RAS pathway-mutated ALL. We, therefore, preclinically evaluated the combination of dexamethasone and selumetinib in vitro and in an orthotopic mouse model engrafted with primary-derived ALL cells and showed pronounced drug synergism in RAS pathway-mutated ALL. These data suggest that this drug combination may be highly effective in the significant subgroup of patients with this form of leukemia and has led to the Seludex trial, an international phase I/II expansion study on the treatment of relapsed/refractory RAS pathway-mutated ALL.

\section{Methods}

\section{Compounds and formulation}

Selumetinib was kindly provided by AstraZeneca (Cheshire, UK). For the in vitro studies, it was dissolved in dimethylsulfoxide to a concentration of $100 \mathrm{mM}$ and stored in single-use aliquots at $-20^{\circ} \mathrm{C}$. Dexamethasone was purchased from Sigma-Aldrich (Dorset, UK), dissolved in ethanol at $20 \mathrm{mM}$ and stored at $-20^{\circ} \mathrm{C}$. For in vivo studies, selumetinib was prepared as a suspension in $0.5 \%$ hydroxypropyl methylcellulose $+0.1 \%$ polysorbate 80 .

\section{Patients' samples}

Primagrafts were generated in NOD SCID $\gamma$ null (NSG) mice using ALL cells from bone marrow samples of children presenting or relapsing with ALL and accessed through the Newcastle Haematology Biobank, after appropriate consent (reference numbers 2002/111 and 07/H0906). Clinical details of the patients are given in Table 1. Mutational screening for RAS pathway mutations and assessment of pathway activation by western blotting of p-ERK was performed as previously described. ${ }^{8,23}$

\section{In vitro drug sensitivity and synergy}

Freshly harvested primagraft cells were suspended in RPMI1640 with $15 \%$ fetal bovine serum and plated out in triplicate at a density of $5 \times 10^{5}$ cells $/ 100 \mu \mathrm{L} /$ well into 96 -well plates and treated with a range of concentrations of dexamethasone $(0.1 \mathrm{nM}$ to $10 \mu \mathrm{M})$ or selumetinib ( $1 \mathrm{nM}$ to $100 \mu \mathrm{M})$. After $96 \mathrm{~h}$, cytotoxicity was assessed using the CellTiter 96 Aqueous One kit (Promega, Southampton, UK). The results were averaged and expressed as a percentage of the cytotoxicity of the control vehicle. Survival curves were plotted and half maximal growth inhibitory values $\left(\mathrm{GI}_{50}\right)$ were calculated using GraphPad Prism software (GraphPad software Inc., San Diego, CA, USA). Drug combination experiments were analyzed for synergistic, additive, or antagonistic effects using the combination index method developed by Chou and Talalay. ${ }^{24}$ Briefly, primagraft cells were treated with fixed dose ratios based on the $\mathrm{GI}_{50}$ values of each drug ( $\mathrm{x} 0.25, \mathrm{x} 0.5, \mathrm{x} 1, \mathrm{x} 2$ and $\mathrm{x} 4)$ and evaluated by median effect analysis using CalcuSyn software (Cambridge, UK). The dose-effect curve for each drug alone

Table 1. Clinical features of patients and characterization of patient-derived xenografts.

\begin{tabular}{|c|c|c|c|c|c|c|c|}
\hline Patient ID & Sex & $\begin{array}{l}\text { Age at diagnosis } \\
\text { (years) }\end{array}$ & Cytogenetics & $\begin{array}{c}\text { End of induction } \\
\text { MRD }\end{array}$ & $\begin{array}{l}\text { Ras pathway } \\
\text { mutation }\end{array}$ & Clonality & pERK \\
\hline L779 & M & 5.5 & High hyperdiploid & Intermediate & NRAS (Q61R) & Clonal & Positive \\
\hline $\mathrm{L}^{897^{\mathrm{a}}}$ & M & 16.8 & B other & High risk & $K R A S$ (G12D) & Clonal & Positive \\
\hline L914 & $\mathrm{F}$ & 7.3 & High hyperdiploid & Low risk & $\begin{array}{c}\text { CBL/FLT3 } \\
\text { large del/ } / \Delta 836\end{array}$ & Clonal & Positive \\
\hline L829b relapse & $\mathrm{F}$ & 3.1 & High hyperdiploid & High risk & KRAS (G13D) & Clonal & Positive \\
\hline L707 & $\mathrm{F}$ & 16.5 & $\mathrm{t}(17 ; 19)$ & High risk & $K R A S$ (insertion) & Clonal & Positive \\
\hline LX825 & $\mathrm{F}$ & 14.7 & B other & High risk & Wildtype & N/A & Negative \\
\hline L920 & $\mathrm{F}$ & 4.4 & B other & Low risk & Wildtype & N/A & Negative \\
\hline L848 & M & 2.5 & $\mathrm{t}(12 ; 21)$ & Low risk & Wildtype & N/A & Negative \\
\hline
\end{tabular}

${ }^{a}$ Patient suffered an on-treatment central nervous system relapse. ${ }^{\circ}$ L829 at diagnosis was NRAS G12D. ${ }^{~}$ Patient relapsed with the same $K R A S$ mutation. B-other group:-; L897 is negative by fluorescence in situ hybridization (FISH) for ETV6-RUNX1, BCR-ABL1, MLL and TCF3-PBX1/HLF. LX825 is negative by FISH for ETV6-RUNX1, BCR-ABL1, MLL, CRLF2, IKZF1,PAX5, IGH and PDGFRB. ID: identity; MRD: minimal residual disease; M: male; F: female; N/A: not available. 
was determined using the median-effect principle and was compared to the effect achieved with a combination of the two drugs to derive a combination index (CI) value.

\section{Pharmacokinetic analyses}

Plasma pharmacokinetics of selumetinib and dexamethasone were determined using non-compartmental analysis in female CD1 mice after oral dosing. Plasma concentrations of both drugs were measured by liquid chromatographic mass spectrometry (API4000 LCMS/MS, Applied Biosystems, CA, USA), attached to a Perkin Elmer chromatography system (Perkin Elmer Ltd, Beckonsfield, UK) and calibrated using standards prepared in blank mouse plasma. In both cases separation was performed using a Gemini $3 \mu$ C18 110A column, (50x3 mm), fitted with a 4x2 mm C18 cartridge (Phenomenex, Macclesfield, UK.

\section{In vivo experiments}

All experiments were performed under the UK Home Office NCL-PLL60/4552. Drug efficacy studies were performed as previously described. ${ }^{6}$ Briefly, primagraft cells were injected intrafemorally and mice were monitored for engraftment every 34 weeks by tail vein bleed. Blood red cells were lysed and analyzed by flow cytometry on a BD FACSCanto II, using antihuman CD10, CD34 and CD19 and anti-mouse CD45 antibodies. Human leukemia cells were gated and their number expressed as a percentage of the total number of nucleated cells. Once the level of human leukemia cells reached $>1 \%$ of total cells, mice were randomized into groups to receive control vehicle $(0.5 \%$ hydroxypropyl methylcellulose $+0.1 \%$ polysorbate 80 ) or drug treatment (6 mice per group) with dexamethasone, selumetinib or both, administered by oral gavage. Selumetinib was dosed at $25 \mathrm{mg} / \mathrm{kg}$ bid, while the dexamethasone dose varied in each study. Tumor burden was monitored weekly by flow cytometry. Pharmacodynamic studies were performed in highly engrafted mice which were dosed for $72 \mathrm{~h}$. Spleens were removed following euthanasia and assessed by flow cytometry to confirm an engraftment of $>85 \%$. Cells were lysed and analyzed by western blotting for levels of p-ERK, ERK2, BIM, MCL1 and $\alpha$-tubulin, as described above.

Additional details of the study methods are provided in the Online Supplementary Material.

\section{Results}

The combination of selumetinib and dexamethasone show synergy in vitro in RAS pathway-mutated acute lymphoblastic leukemia and is associated with enhanced induction of BIM

To investigate possible synergism, the R3F9 cell line and primagraft ALL cells, with and without Ras pathway mutations $(n=8)$, were treated with dexamethasone,
A

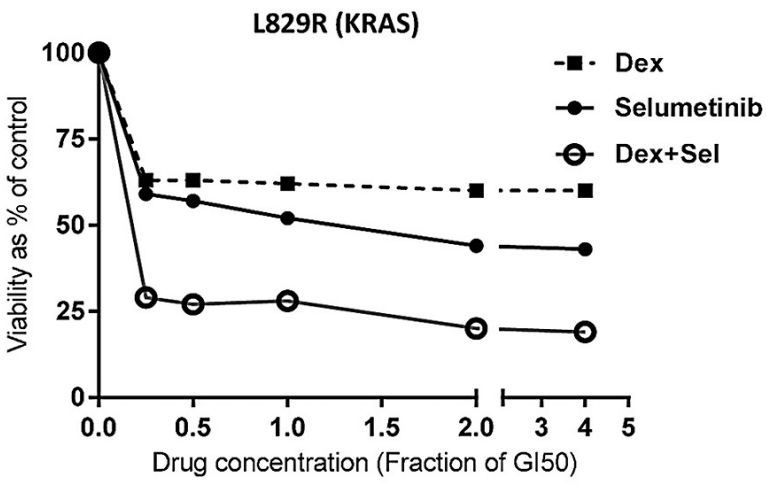

C

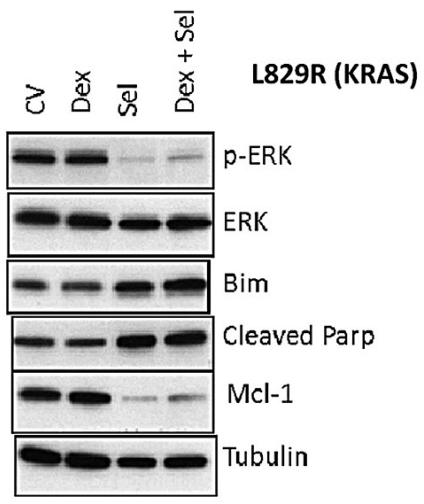

B

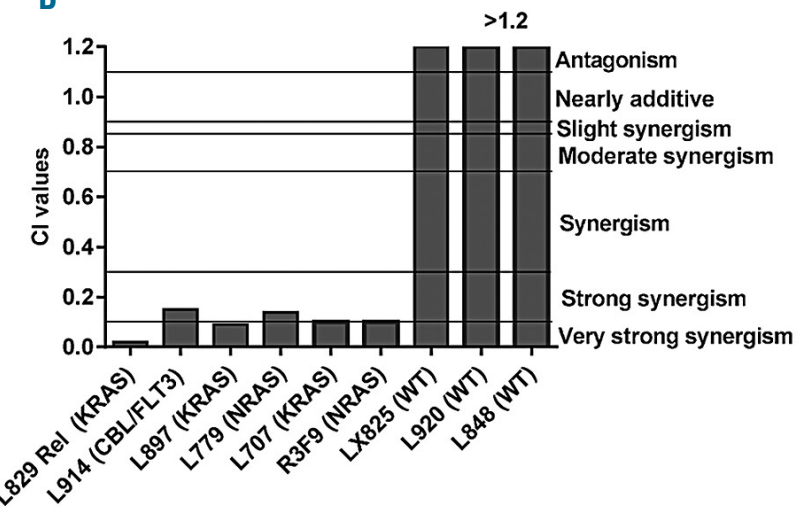

D

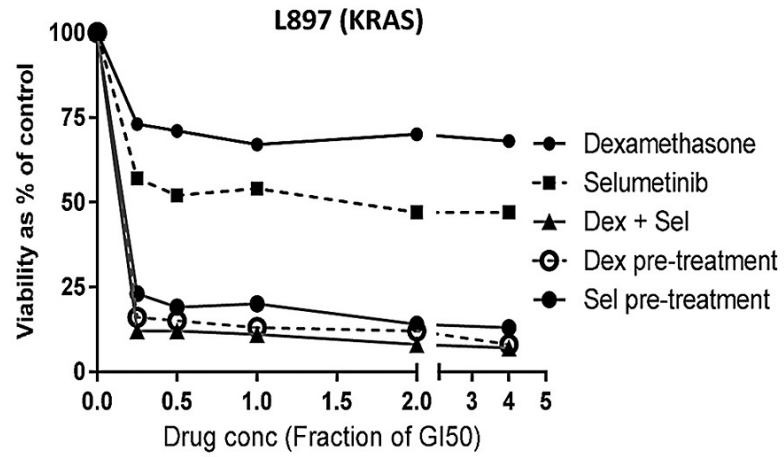

Figure 1. The combination of selumetinib and dexamethasone shows synergy in vitro in RAS pathway-mutated acute lymphoblastic leukemia and is associated with enhanced levels of BIM. (A) Viability curves of Ras pathway-mutant acute lymphoblastic leukemia (ALL) cells (L829R) with individual drugs and the selumetinib/dexamethasone drug combination. (B) Histogram of combination indices for the selumetinib/dexamethasone combination in wildtype and Ras pathway-mutant

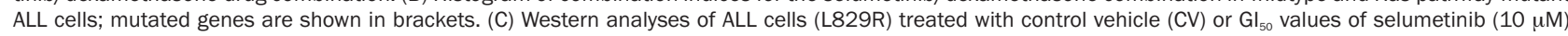
and dexamethasone $(10 \mu \mathrm{M})$, singly and in combination. (D) A representative median effect curve (data shown are from L897) after simultaneous drug dosing and with each drug added $24 \mathrm{~h}$ prior to the partner drug, followed by a further $72 \mathrm{~h}$ incubation. CV: control vehicle; Sel: selumetinib; Dex: dexamethasone; Cl: combination index. 
selumetinib and the drug combination at $0.25 \mathrm{x}, 0.5 \mathrm{x}, 1 \mathrm{x}$, $2 \mathrm{x}$ and $4 \mathrm{x}$ their respective $\mathrm{GI}_{50}$ concentrations and viability data were evaluated by median effect analysis. The CI for all RAS pathway-mutated samples were indicative of strong synergy with a mean of 0.1 (range, 0.02-0.15) (Figure 1A,B and Online Supplementary Figure S1). Synergism was not observed in ALL primagraft cells without RAS pathway activation $(\mathrm{CI}>1.2)$. Mechanistic assessments were performed with the $\mathrm{GI}_{50}$ concentration of both drugs for $24 \mathrm{~h}$. As expected, ALL cells treated with selumetinib resulted in almost complete inhibition of ERK phosphorylation and downregulation of MCL1 levels. Dexamethasone treatment also downregulated pERK levels. Treatment with dexamethasone or selumetinib was associated with increased levels of BIM which were further enhanced with the drug combination. A representative western blot and a histogram of the combined densitometry values ( $\mathrm{n}=4$ patient-derived xenografts) are shown in Figure 1C and Online Supplementary Figure S2A. The apoptotic marker, cleaved PARP, was enhanced with the drug combination in some, but not all, patient-derived xenograft samples at this time point (Online Supplementary Figure S2B). While the loss in cell viability in non-dividing patient-derived xenograft ALL cells must be due to increased cell death, we also showed enhanced apoptosis with the drug combination for the NRAS-mutated R3F9 cell line (Online Supplementary Figure S2C,D), an effect reduced by BIM knockdown (Online Supplementary Figure $S 2 E, F)$. There were similar levels of induction of the glucocorticoid receptor target gene, GILZ, in cells treated with both dexamethasone and with the drug combination suggesting that enhanced glucocorticoid receptor transcriptional activity is not a component of the synergism (Online Supplementary Figure S3). Synergism between selumetinib and other drugs for example, gemcitabine, is highly schedule dependent and sequential rather than simultaneous dosing appears optimal. ${ }^{25}$ Thus, we assessed synergism in primagraft ALL cells dosed simultaneously or with only selumetinib or dexamethasone for $24 \mathrm{~h}$ followed by both drugs for an additional $72 \mathrm{~h}$, prior to cell viability assessments. We saw similar synergism across all experimental parameters (Figure 1D) and thus we selected simultaneous drug administration in subsequent in vivo studies.

\section{Pharmacokinetic studies to define a clinically relevant oral dose and exclude drug-drug interactions}

To determine the optimal oral dose of dexamethasone that will achieve clinically relevant serum levels, pharmacokinetic studies were performed in CD1 mice. Mice $(\mathrm{n}=27)$ were dosed with $0.5,1$ and $5 \mathrm{mg} / \mathrm{kg}$ dexamethasone by oral gavage. Blood samples were taken after 15 min, 30 min, $1 \mathrm{~h}, 3 \mathrm{~h}, 6 \mathrm{~h}$ and $24 \mathrm{~h}$ and serum dexamethasone levels were analyzed (Online Supplementary Figure S4A). A $\mathrm{T}_{\max }$ of 60 min was observed, with $\mathrm{C}_{\max }$ values of 48.9, 94.7 and $766.5 \mathrm{ng} / \mathrm{mL}$ following the 0.5, 1 and 5 $\mathrm{mg} / \mathrm{kg}$ doses, respectively. Given the reported $\mathrm{C}_{\max }$ average of $40-90 \mathrm{ng} / \mathrm{mL}$ in recent UK and American ALL trials, 1 $\mathrm{mg} / \mathrm{kg}$ was deemed the most appropriate dose level..$^{26,27}$

Dexamethasone can induce cytochrome P450 forms, including CYP3A4, the principal isoform responsible for selumetinib oxidative metabolism; we therefore performed selumetinib pharmacokinetic analyses, following administration of the drug alone $(25 \mathrm{mg} / \mathrm{kg})$ and after coadministration with $1 \mathrm{mg} / \mathrm{kg}$ dexamethasone (Online
Supplementary Figure $S 4 B, C) . A \mathrm{~T}_{\max }$ of $60 \mathrm{~min}$ was observed, with $C_{\max }$ values for selumetinib of $4.74 \mu \mathrm{g} / \mathrm{mL}$ compared to $5.49 \mu \mathrm{g} / \mathrm{mL}$, respectively $(P>0.05$, Student $t$ test). Other parameters were also similar (Online Supplementary Figure S4C), indicative of no drug-drug interaction $(P>0.05$ for all).

\section{Selumetinib and dexamethasone show synergy in vivo and clear central nervous system disease}

The drug combination was evaluated in vivo and its effects compared to those of single drugs and control vehicle in primagrafts derived from diagnostic ALL (NRAS Q61R and KRAS G12D) and relapse (KRAS G13D) samples. Scheduling and dosing, by oral gavage, are shown in Figure 2A-G. Given the significant weight loss (>20\%) associated with dexamethasone, dosing could not be prolonged, even when the dose was lowered from $1 \mathrm{mg} / \mathrm{kg}$ bid to 0.25 $\mathrm{mg} / \mathrm{kg}$ sid. There was no additional toxicity observed in mice given the drug combination. Nevertheless, at the end of the dosing period, there was a significant reduction in spleen size with selumetinib or dexamethasone alone but a statistically greater reduction in mice given the drug combination, with spleen weights approaching those of healthy mice $(P<0.001)$ (combined data are shown in Figure $2 \mathrm{G}$ ). In addition, brains were assessed for the depth of leukemia infiltration in the leptomeninges. For mice engrafted with L897 and L779 primagraft cells, there was a significant reduction in leukemic infiltration in drug-treated mice, with a mean and standard deviation of $66.3 \mu \pm 100.6$ for animals given the control vehicle, compared to $3.1 \mu \pm 12.5$ for those treated with dexamethasone and $5.37 \mu \pm 21.475$ for those treated with selumetinib (Online Supplementary Figure S5A). Mice treated with the drug combination showed no leukemic infiltration $(P<0.05$ for all by the Student $t$ test). For L779, there was demonstrable CNS disease once peripheral ALL exceeded 1\% i.e. pre-dosing (Online Supplementary Figure S5B). Clearance of CNS disease in mice engrafted with L829R cells was unevaluable because of minimal CNS leukemia in both control and drug-treated mice. The results of the pharmacodynamic assessment of engrafted spleens after short-term dosing were consistent with observations in vitro; inhibition of ERK phosphorylation and lower MCL1 levels associated with selumetinib dosing, similar induction of GILZ with dexamethasone dosing, and modest enhancement of BIM levels with the drug combination (Figure $3 \mathrm{~A}-\mathrm{D})$. Annexin $\mathrm{V}$ binding in circulating ALL cells, as detected by multi-parameter flow cytometry, increased at both $24 \mathrm{~h}$ and $48 \mathrm{~h}$ in all drug-treated mice and was highest for those given the drug combination (Figure 3E).

\section{Discussion}

Selumetinib is a potent, selective, allosteric inhibitor of MEK1/2 with demonstrated anti-tumor activity and a favorable toxicity profile. It has progressed to phase III clinical trials for several types of adult solid cancers. ${ }^{28-30}$ In the pediatric setting, selumetinib has recently undergone phase I clinic testing as a monotherapy in children with $B R A F$-driven recurrent/refractory pediatric low-grade glioma which defined a maximum tolerated dose of 25 $\mathrm{mg} / \mathrm{m}^{2} /$ dose bid. $^{31}$ Sustained responses (1 complete, 7 partial) were observed in some children and selumetinib was well tolerated, with the most common toxicity being rash. 
A

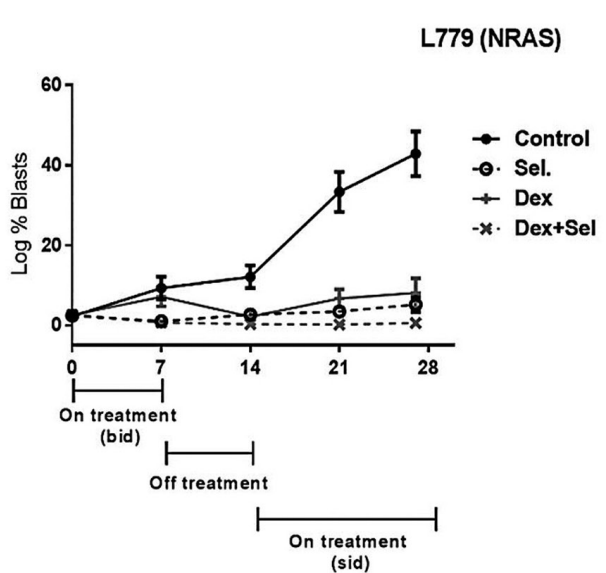

C

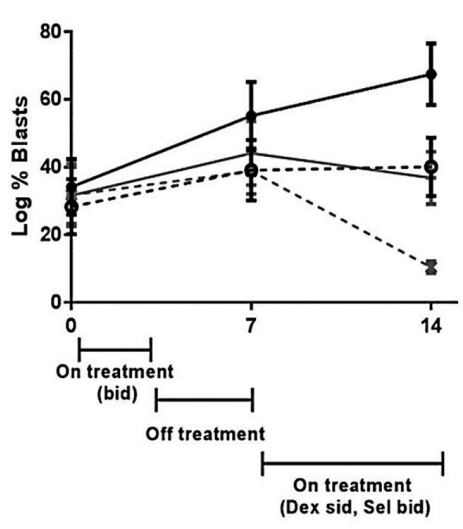

$E$

897 (KRAS)

$\rightarrow$ control

-๑. Sel

+ Dex

-x. Dex+Sel

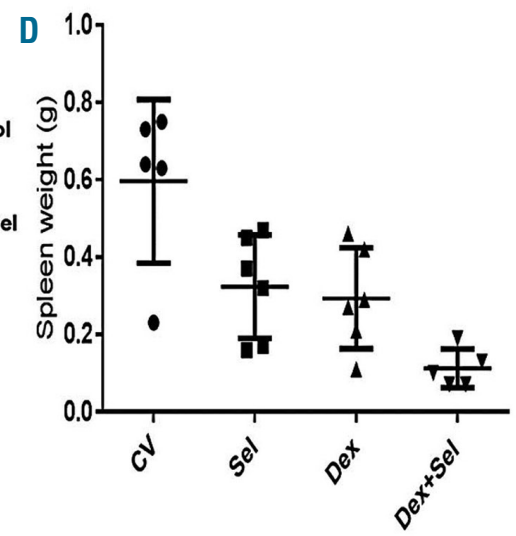

$F$

L829R (KRAS)
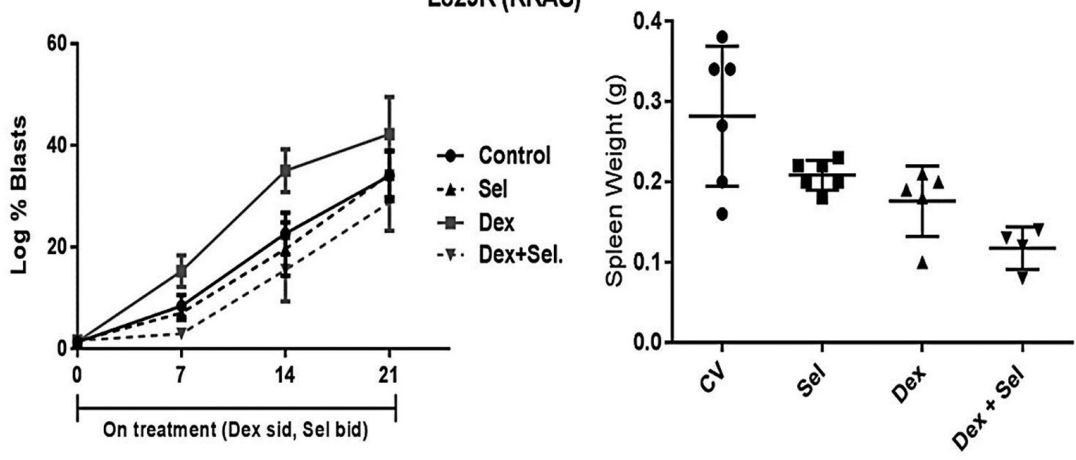

B

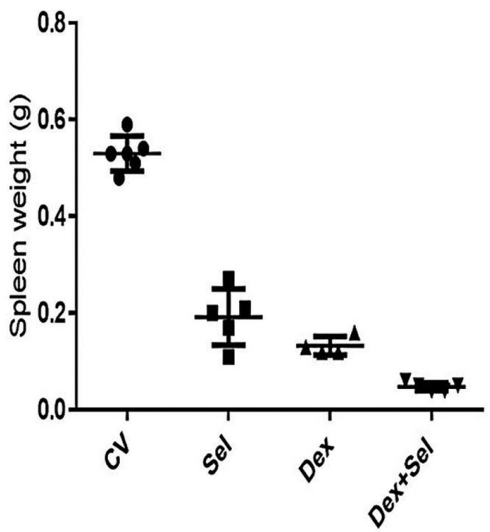

$\rightarrow$ Control

- - Sel

$\rightarrow-\operatorname{Dex}$

- r. Dex+Sel.
G

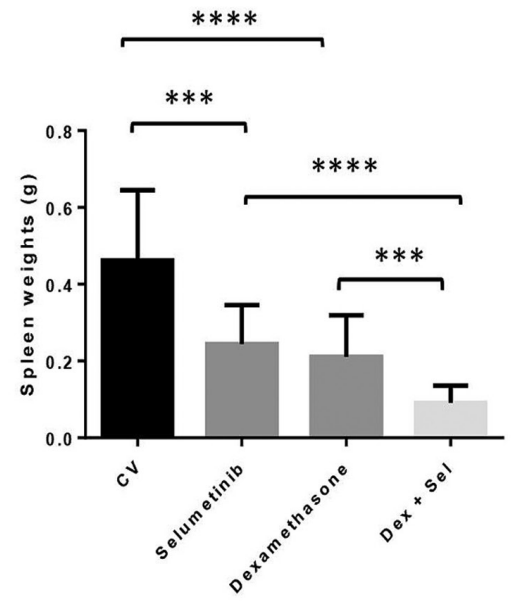

Figure 2. Selumetinib and dexamethasone show synergy in vivo in RAS pathwaymutated acute lymphoblastic leukemia. (A-F) In vivo drug efficacy studies of single drugs and their combination in RAS pathway mutated-acute lymphoblastic leukemia (ALL) showing dose scheduling and peripheral blood monitoring before and during dosing and spleen weights at the end of dosing for mice with L779-NRAS (A and B, respectively), L897-KRAS (C and D) and L829 relapse- KRAS (E and F) ALL. For L779, mice were dosed with selumetinib at $25 \mathrm{mg} / \mathrm{kg}$ and dexamethasone at 1 $\mathrm{mg} / \mathrm{kg}$ twice daily and then once daily after a recovery period. For L897, the dosage of selumetinib was $25 \mathrm{mg} / \mathrm{kg}$ and that of dexamethasone $0.5 \mathrm{mg} / \mathrm{kg}$ (bid), with the dexamethasone being increased to $1 \mathrm{mg} / \mathrm{kg}$ (sid) following a recovery period. For L829R, selumetinib was dosed at $25 \mathrm{mg} / \mathrm{kg}$ (bid) and dexamethasone at 0.25 $\mathrm{mg} / \mathrm{kg}$ (sid). (G) The mean and standard deviation are shown for combined spleen weight data for all three efficacy experiments (one-way analysis of variance with the Tukey multiple comparison test, $* * * P<0.001, * * * * P<0.0001 ; n=17$ mice treated with control vehicle, $n=17$ treated with selumetinib, $n=15$ treated with dexamethasone and $n=14$ treated with the combination. CV: control vehicle; Sel: selumetinib; Dex: dexamethasone. 


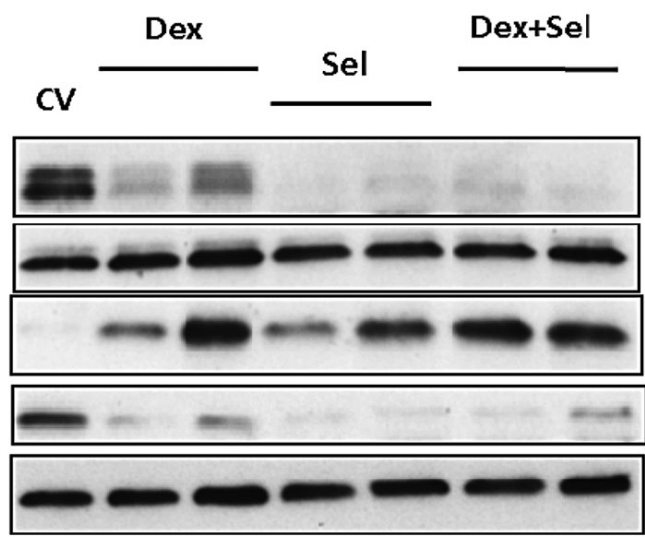

p-ERK

ERK

Bim

Mcl-1

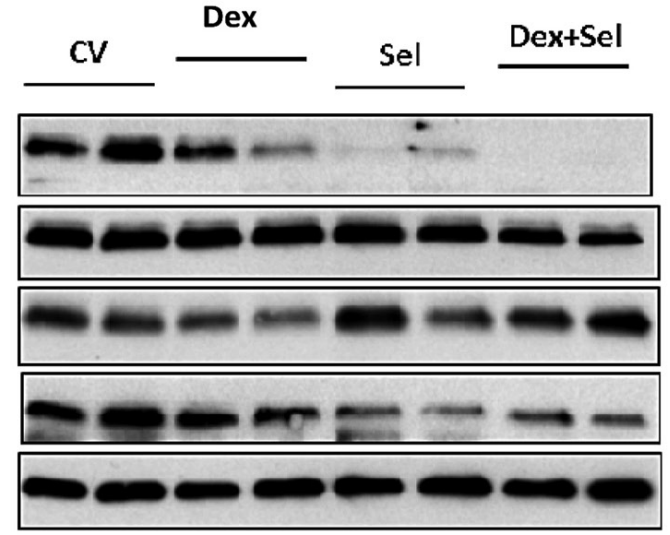

p-ERK

$\alpha$-Tubulin

C

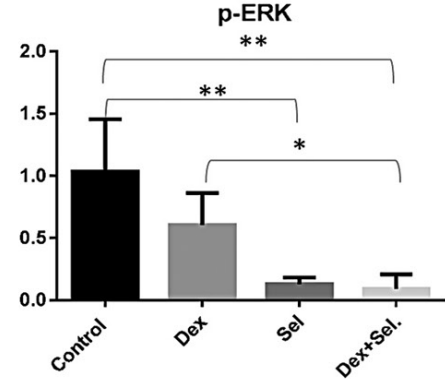

BIM

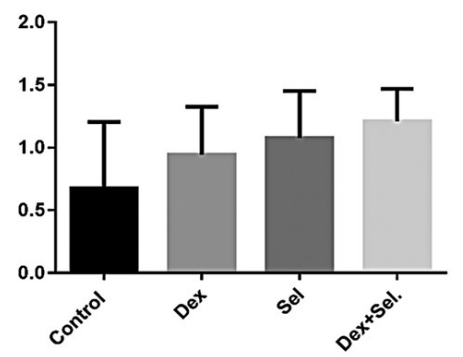

D

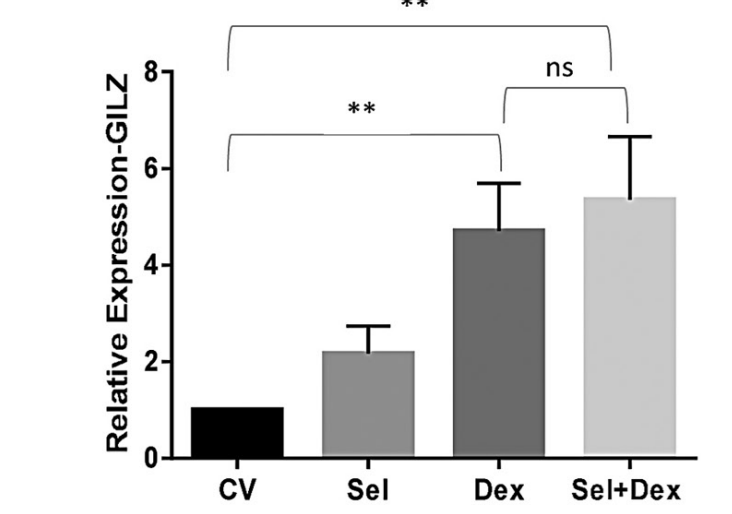

ERK
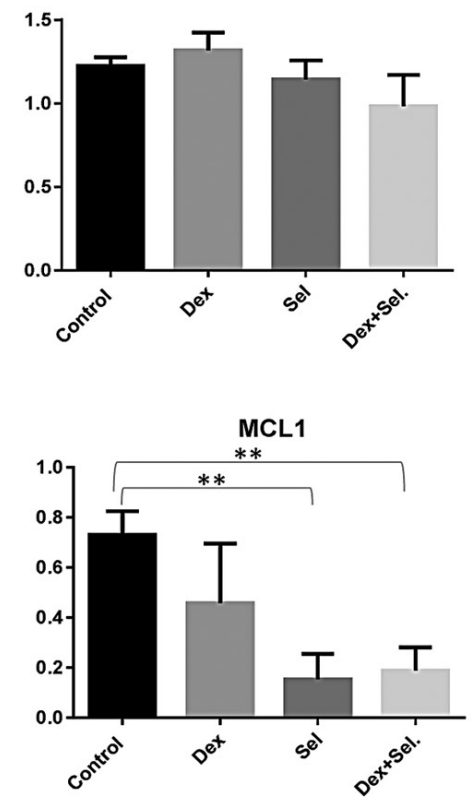

E

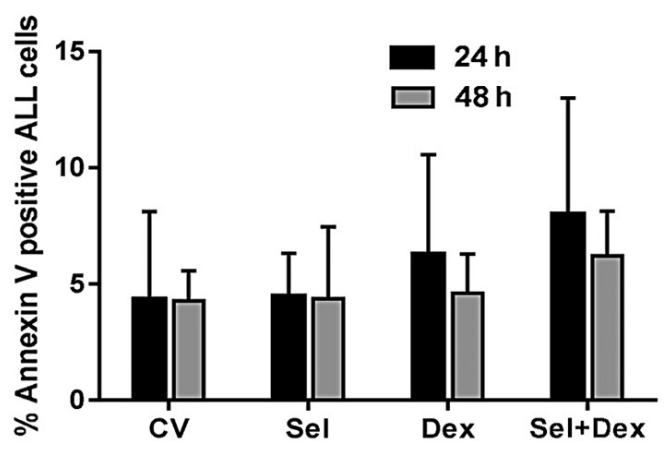

Figure 3. Pharmacodynamic analyses in acute lymphoblastic leukemia cells after drug dosing in vivo support in vitro data. (A,B) Western blot analyses of spleen cells from mice engrafted with RAS pathway-mutant acute lymphoblastic leukemia cells $72 \mathrm{~h}$ after dosing: L779; NRAS; $25 \mathrm{mg} / \mathrm{kg}$ selumetinib and $1 \mathrm{mg} / \mathrm{kg}$ dexamethasone bid (A) and L897; KRAS; $25 \mathrm{mg} / \mathrm{kg}$ selumetinib and $0.5 \mathrm{mg} / \mathrm{kg}$ dexamethasone bid (B). (C) Histograms of densitometry from western blot analyses, showing mean \pm standard error of mean (SEM) (3-4 mice per treatment) [one-way analysis of variance (ANOVA) with the Tukey multiple comparison test, * $P<0.05, * * P<0.01$ ] (D) Relative expression of GILZ mRNA (mean and SEM) in treated mice compared to those given the control vehicle, as quantified by real-time polymerase chain reaction analysis in all three patient-derived xenograft experiments, again after $72 \mathrm{~h}$ dosing (ANOVA as before **P<0.01; ns, not significant. (E) Histograms of annexin V-positive ALL cells (mean \pm SEM) determined by flow cytometric analyses of peripheral blood at $24 \mathrm{~h}$ and $48 \mathrm{~h}$ after dosing ( $2 \mathrm{mice}$ per group). CV: control vehicle; Sel: selumetinib; Dex: dexamethasone. 
In addition, a phase I trial of selumetinib in children with neurofibromatosis type 1 and inoperable plexiform neurofibromas showed partial responses in 17 of 24 children $(71 \%)$ and no excess toxicity was reported. ${ }^{32}$

While we have previously shown preclinical activity of selumetinib in ALL, this drug, like other MEK inhibitors, is likely to show maximal therapeutic benefit in combination. Indeed, in phase III clinical trials for advanced nonsmall-cell lung cancer and uveal melanoma, selumetinib has been evaluated in combination with docetaxel and dacarbazine, respectively. ${ }^{33}$ In this current study, we show significant synergy of selumetinib with the synthetic glucocorticoid dexamethasone in vitro and in an orthotopic mouse model engrafted with RAS pathway-activated primary-derived ALL cells. Importantly, we demonstrate this across a range of cytogenetic subgroups, including high hyperdiploidy, B-other, $\mathrm{t}(17 ; 19)$ and $\mathrm{t}(1 ; 19)$ ALL. Pharmacokinetic data showed clinically relevant drug levels and optimal scheduling and in vivo pharmacodynamic analyses confirmed an impact on drug targets and apoptosis. Mechanistically, the synergism was associated with enhanced induction of the pro-apoptotic protein, BIM, and decreases in the anti-apoptotic BH3-only protein, MCL1. BIM is a BH3-only protein that binds to anti-apoptotic BCL2 family members, including MCL1 and BCL2, to liberate and directly activate bax and bak, which then elicit caspase-dependent apoptosis. BIM is an effector protein in both glucocorticoid and MEK inhibitor responses and reducing $\mathrm{pERK}$ activity enhances BIM levels as well as decreasing MCL1 protein levels by increasing its turnover. ${ }^{34}$ Therefore, we propose that the drug combination enables BIM to inhibit anti-apoptotic BH3-only proteins more completely and directly activate BAX and BAK. Others have reported a direct effect of MEK inhibition on glucocorticoid receptor transcriptional activity which may also contribute to the synergism, but this did not appear significant in our experiments at the time point chosen. ${ }^{22}$ Epigenetic regulation of the BIM locus due to acetylation has been described in a subgroup of glucocorticoid-resistant ALL and is associated with BIM under-expression. Such individuals may be expected to have a suboptimal response to the selumetinib/dexamethasone drug combination. ${ }^{35}$ However, the incidence of acetylated BIM in the relapsed setting and in the context of RAS pathway mutations has not been described to date. Our synergism data are supported by a study by Jones et al., who used an integrated approach to understand glucocorticoid resistance and relapse and identified MAPK pathways as a contributory factor. ${ }^{22}$ In this study, knockdown of MEK2 or MEK inhibition enhanced responses not only to glucocorticoids but also to other chemotherapeutics and was not dependent on the presence of RAS pathway mutations, a finding suggestive of activation of the pathway through alternative routes. We have previously shown an excellent correlation between pERK activation and the presence of RAS pathway mutations, although we too noted some rare exceptions which in our study were in part explained by the presence of chromosomal translocations, including the Philadelphia chromosome and 11q23. ${ }^{\circ}$

Drug synergies have also been shown for MEK inhibitors with both traditional chemotherapeutics such as gemcitabine and targeted agents including PI3K/AKT inhibitors ${ }^{36,37}$ and the BCL-XL inhibitor, navitoclax (ABT263). ${ }^{38}$ Inhibiting the other effector pathways of RAS is clearly a rational strategy; however, while we have observed synergism of MEK and AKT inhibition in RAS pathway-mutated ALL in vitro, the synergism was considerably weaker than that observed with dexamethasone (unpublished observations). In solid cancers, increased levels of BIM protein are also observed with MEK inhibition, but the protein is inactive due to sequestration by high levels of BCL-XL. In the presence of navitoclax, BIM is released, triggering an apoptotic response. ${ }^{38}$

We have previously reported a reduction of CNS leukemia in selumetinib-treated mice and now confirm this in additional primagraft samples and show complete absence of leukemic infiltration in the leptomeninges of mice treated with the selumetinib/dexamethasone drug combination. ${ }^{6}$ The identification of CNS disease in mice with similar levels of ALL engraftment prior to drug dosing suggests that the drug combination completely eradicated the leukemia in situ. This is a highly significant finding given the association of RAS pathway mutations and CNS disease at relapse that we previously reported in the IBFMREZ2002 clinical trial and the fact that in contemporary regimens, the proportion of CNS relapses is increasing. ${ }^{39}$

A key question, relevant to MEK inhibitor therapy, is whether Ras pathway mutations are initiating events in ALL or secondary, cooperating genetic events and there is evidence for both. ${ }^{13}$ However, for targeted therapies to be successful, the target is ideally present on all tumor cells and we and others have reported that mutations can be subclonal, particularly at diagnosis, and can be gained or lost at relapse. ${ }^{6,40-42}$ Importantly, we have also shown that mutations at relapse are in the major ALL clone, are often selected from a minor subclone at diagnosis and that apparent 'loss' of a Ras pathway mutation can be 'replacement' of one for another. ${ }^{6,8,43}$ This suggests a dependence on the pathway that can be exploited by MEK inhibition and, as we show here, is enhanced with co-exposure to dexamethasone.

Based on these promising data, an international phase I/II clinical trial of oral dexamethasone and selumetinib (Seludex) is underway in RAS pathway-mutated, multiply relapsed/refractory ALL. A parallel, national study in adult disease at first relapse is also ongoing, since the prevalence of RAS pathway mutations and association with poor prognosis has also been noted..$^{44}$ One relevant observation from selumetinib and other Mek inhibitor trials is that the most common toxicity is inflammatory rash. In severe cases, the recommended treatment is oral glucocorticoids and no adverse effects of drug co-administration have been reported. ${ }^{45}$ Thus, if efficacy is seen in the proposed clinical trials, selumetinib and other Mek inhibitors may be a much needed novel therapy for a substantial number of children with high-risk, relapsed disease. There may also be a role for the drug combination in the upfront treatment of RAS-driven, high-risk ALL, to avert relapse.

\section{Acknowledgments}

The authors gratefully acknowledge Cancer Research UK (project grant to JAEI, HN and JV, number 18780), Bloodwise (previously known as the Leukaemia and Lymphoma Research Fund, project grant to JAEI, number 11007), the North of England Children's Cancer Research Fund and the Newcastle Haematology Biobank for ALL samples. We are grateful to AstraZeneca for their kind donation of selumetinib. CH is funded by the Chief Scientist Office (ETM/374). We thank Clare Orange and Lynn Stevenson, University of Glasgow and Think Pink, Scotland for help with histology and slide scanning. 


\section{References}

1. Vora A, Goulden N, Mitchell C, et al. Augmented post-remission therapy for a minimal residual disease-defined high-risk subgroup of children and young people with clinical standard-risk and intermediate-risk acute lymphoblastic leukaemia (UKALL 2003): a randomised controlled trial. Lancet Oncol. 2014:15(8):809-818.

2. Vora A, Goulden N, Wade R, et al. Treatment reduction for children and young adults with low-risk acute lymphoblastic leukaemia defined by minimal residual disease (UKALL 2003): a randomised controlled trial. Lancet Oncol. 2013:14(3):199-209.

3. Parker C, Waters R, Leighton C, et al. Effect of mitoxantrone on outcome of children with first relapse of acute lymphoblastic leukaemia (ALL R3): an open-label randomised trial. Lancet. 2010;376(9757):2009-2017.

4. Hof J, Krentz S, van Schewick C, et al. Mutations and deletions of the TP53 gene predict nonresponse to treatment and poor outcome in first relapse of childhood acute lymphoblastic leukemia. J Clin Oncol. 2011;29(23):3185-3193

5. Malempati S, Gaynon PS, Sather H, La MK, Stork LC, Children's Oncology Group. Outcome after relapse among children with standard-risk acute lymphoblastic leukemia: Children's Oncology Group study CCG1952. J Clin Oncol. 2007;25(36):5800-5807.

6. Irving I, Matheson E, Minto L, et al. Ras pathway mutations are prevalent in relapsed childhood acute lymphoblastic leukemia and confer sensitivity to MEK inhibition. Blood. 2014;124(23):3420-3430

7. Moorman AV, Irving J, Enshaei $\mathrm{A}$, et al. Composite index for risk prediction in relapsed childhood acute lymphoblastic Leukaemia. Haematologica. 2015;100(s1): 195-196.

8. Case M, Matheson E, Minto L, et al. Mutation of genes affecting the RAS pathway is common in childhood acute lymphoblastic leukemia. Cancer Res. 2008;68 (16):6803-6809

9. Chung E, Kondo M. Role of Ras/Raf/MEK/ERK signaling in physiological hematopoiesis and leukemia development. Immunol Res. 2011:49(1-3):248-268.

10. Pylayeva-Gupta Y, Grabocka E, Bar-Sagi D. RAS oncogenes: weaving a tumorigenic web. Nat Rev Cancer. 2011;11(11):761-774.

11. Ahearn IM, Haigis K, Bar-Sagi D, Philips MR. Regulating the regulator: post-translational modification of RAS. Nat Rev Mol Cell Biol. 2012;13(1):39-51.

12. Ward AF, Braun BS, Shannon KM. Targeting oncogenic Ras signaling in hematologic malignancies. Blood. 2012;120(17):33973406.

13. Knight T, Irving JA. Ras/Raf/MEK/ERK Pathway activation in childhood acute lymphoblastic leukemia and its therapeutic targeting. Front Oncol. 2014; 4:160.

14. Balmanno K, Cook SJ. Tumour cell survival signalling by the ERK1/2 pathway. Cell Death Differ. 2009;16(3):368-377.

15. Ley R, Ewings KE, Hadfield K, Cook SJ. Regulatory phosphorylation of Bim: sorting out the ERK from the JNK. Cell Death Differ. 2005;12(8):1008-1014

16. Meng J, Fang B, Liao Y, Chresta CM, Smith PD, Roth JA. Apoptosis induction by MEK inhibition in human lung cancer cells is medi- ated by Bim. PLoS One. 2010;5(9):e13026.

17. Hongo T, Fujii Y. In vitro chemosensitivity of lymphoblasts at relapse in childhood leukemia using the MTT assay. Int J Hematol. 1991;54(3):219-230

18. Klumper E, Pieters R, Veerman AJ, et al. In vitro cellular drug resistance in children with relapsed/refractory acute lymphoblastic leukemia. Blood. 1995;86(10):3861-3868.

19. Lu J, Quearry B, Harada H. p38-MAP kinase activation followed by BIM induction is essential for glucocorticoid-induced apoptosis in lymphoblastic leukemia cells. FEBS Lett. 2006:580(14):3539-3544

20. Rambal AA, Panaguiton ZL, Kramer L, Grant S, Harada H. MEK inhibitors potentiate dexamethasone lethality in acute lymphoblastic leukemia cells through the proapoptotic molecule BIM. Leukemia. 2009:23(10):1744-1754

21. Polak A, Kiliszek P, Sewastianik T, et al. MEK inhibition sensitizes precursor B-cell acute lymphoblastic leukemia (B-ALL) cells to dexamethasone through modulation of mTOR activity and stimulation of autophagy. PLoS One. 2016;11(5):e0155893.

22. Jones CL, Gearheart CM, Fosmire S, et al MAPK signaling cascades mediate distinct glucocorticoid resistance mechanisms in pediatric leukemia. Blood. 2015;126(19): 2202-2212

23. Nicholson L, Knight T, Matheson E, et al. Casitas B lymphoma mutations in childhood acute lymphoblastic leukemia. Genes Chromosomes Cancer. 2012;51(3):250 256.

24. Chou TC, Talalay P. Quantitative analysis of dose-effect relationships: the combined effects of multiple drugs or enzyme inhibitors. Adv Enzyme Regul. 1984;22:27-

25. Xu J, Knox JJ, Ibrahimov E, et al. Sequence dependence of MEK inhibitor AZD6244 combined with gemcitabine for the treatment of biliary cancer. Clin Cancer Res. 2013;19(1):118-127.

26. Yang L, Panetta JC, Cai X, et al. Asparaginase may influence dexamethasone pharmacokinetics in acute lymphoblastic leukemia. Clin Oncol. 2008:26(12):1932-1939.

27. Jackson RK, Irving JAE, Veal GJ Pharmacokinetics of standard versus short high-dose dexamethasone therapy in childhood acute lymphoblastic leukemia: results from the UKALL 2011 trial. Cancer Res. 2016;76(14 Suppl):CT115.

28. Bennouna J, Lang I, Valladares-Ayerbes M, et al. A phase II, open-label, randomised study to assess the efficacy and safety of the MEK1/2 inhibitor AZD6244 (ARRY-142886) versus capecitabine monotherapy in patients with colorectal cancer who have failed on or two prior chemotherapeutic regimens. Invest New Drugs. 2011;29(5):1021-1028.

29. Davies BR, Logie A, McKay JS, et al. AZD6244 (ARRY-142886), a potent inhibito of mitogen-activated protein kinase/extracellular signal-regulated kinase kinase 1/2 kinases: mechanism of action in vivo, pharmacokinetic/pharmacodynamic relationship, and potential for combination in preclinical models. Mol Cancer Ther. 2007;6 (8):2209-2219.

30. Janne PA, Shaw AT, Pereira JR, et al. Selumetinib plus docetaxel for KRASmutant advanced non-small-cell lung cancer: a randomised, multicentre, placebo-con- trolled, phase 2 study. Lancet Oncol. 2013;14(1):38-47.

31. Banerjee A, Jakacki R, Onar-Thomas A, et al. A phase 1 study of AZD6244 in children with recurrent or refractory low-grade gliomas: a Pediatric Brain Tumor Consortium report. J Clin Oncol. 2014;32(5s):10065.

32. Dombi E, Baldwin A, Marcus LJ, et al Activity of selumetinib in neurofibromatosis type 1-related plexiform neurofibromas. N Engl J Med. 2016:375(26):2550-2560.

33. Website-https://clinicaltrials.gov/

34. Domina AM, Vrana JA, Gregory MA, Hann SR, Craig RW. MCL1 is phosphorylated in the PEST region and stabilized upon ERK activation in viable cells, and at additional sites with cytotoxic okadaic acid or taxol. Oncogene. 2004;23(31):5301-5315.

35. Bachmann PS, Piazza RG, Janes ME, et al. Epigenetic silencing of BIM in glucocorticoid poor-responsive pediatric acute lymphoblastic leukemia, and its reversal by histone deacetylase inhibition. Blood. 2010;116(16): 3013-3022.

36. Engelman JA, Chen L, Tan X, et al. Effective use of PIBK and MEK inhibitors to treat mutant Kras G12D and PIK3CA H1047R murine lung cancers. Nat Med. 2008;14 (12):1351-1356

37. Haagensen EJ, Kyle S, Beale GS, Maxwell RJ, Newell DR. The synergistic interaction of MEK and PI3K inhibitors is modulated by mTOR inhibition. Br J Cancer. 2012;106(8): 1386-1394

38. Corcoran RB, Cheng KA, Hata AN, et al Synthetic lethal interaction of combined BCL-XL and MEK inhibition promotes tumor regressions in KRAS mutant cance models. Cancer Cell. 2013;23(1):121-128.

39. Krishnan S, Wade R, Moorman AV, et al. Temporal changes in the incidence and pattern of central nervous system relapses in children with acute lymphoblastic leukaemia treated on four consecutive Medical Research Council trials, 1985-2001. Leukemia. 2010;24(2):450-459.

40. Molteni CG, Te Kronnie G, Bicciato S, et al. PTPN11 mutations in childhood acute lymphoblastic leukemia occur as a secondary event associated with high hyperdiploidy. Leukemia. 2010;24(1):232-235

41. Mullighan CG, Zhang J, Kasper LH, et al. CREBBP mutations in relapsed acute lymphoblastic leukaemia. Nature. 2011;471 (7337):235-239.

42. Ma X, Edmonson M, Yergeau D, et al. Rise and fall of subclones from diagnosis to relapse in pediatric B-acute lymphoblastic leukaemia Nat Commun. 2015;6:6604

43. Tartaglia M, Martinelli S, Cazzaniga G, et al Genetic evidence for lineage-related and differentiation stage-related contribution of somatic PTPN11 mutations to leukemogenesis in childhood acute leukemia. Blood. 2004;104(2):307-313

44. Trinquand A, Tanguy-Schmidt A, Ben Abdelali R, et al. Toward a NOTCH1/FBXW7/RAS/PTEN-based oncogenetic risk classification of adult T-cell acute lymphoblastic leukemia: a Group for Research in Adult Acute Lymphoblastic Leukemia study. J Clin Oncol. 2013;31 (34):4333-4342

45. Welsh SI, Corrie PG. Management of BRAF and MEK inhibitor toxicities in patients with metastatic melanoma. Ther Adv Med Oncol. 2015;7(2):122-136. 